\title{
Electrospun polystyrene fibers knitted around imprinted acrylate microspheres as sorbent for paraben derivatives
}

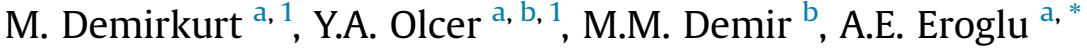 \\ a Department of Chemistry, Izmir Institute of Technology, Urla 35430, Izmir, Turkey \\ b Department of Materials Science and Engineering, Izmir Institute of Technology, Urla 35430, Izmir, Turkey
}

\section{H I G H L I G H T S}

- In this study, a novel SPME fiber has been suggested for a specific recognition sites to parabens.

- In the fiber, a molecularly imprinted polymer microspheres prepared with benzyl paraben as the template was electrospun on the tip of a fiber.

- The fiber has been shown to be selective to the analyte molecules.
G R A P H I C A L A B S T R A C T

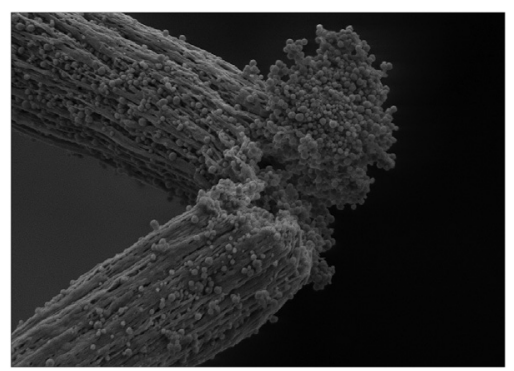

\section{A R T I C L E I N F O}

\section{Article history:}

Received 5 December 2017

Received in revised form

28 January 2018

Accepted 5 February 2018

Available online 14 February 2018

\section{Keywords:}

Molecular imprinted polymers

Solid phase micro-extraction

Electrospinning

High pressure liquid chromatography

Parabens

\begin{abstract}
A B S T R A C T
Parabens are used as antimicrobial preservatives in food, cosmetic products and pharmaceuticals regardless of their endocrine disrupting effect. In this study, highly selective molecular imprinted polymers (MIPs) were synthesized in submicron-sizes and converted to an SPME fiber coating through electrospinning process in order to determine parabens in water samples. Conversion of MIP to a fiber is achieved via creation of spacial knitting around MIP by polystyrene. The selectivity and extraction ability of the fibers were compared with the commercial fibers and the corresponding non-imprinted polymer (NIP) coated fiber. The coated fiber showed better extraction ability among them. Also, the results revealed that the fiber has better selectivity for benzyl paraben and the other structurally-related compounds, such as methyl and propyl paraben. Extraction efficiency of prepared fibers for three parabens has been tested by spiking bottled, tap and sea water samples. The recoveries changed between $92.2 \pm 0.8$ and $99.8 \pm 0.1$ for three different water types. This method could be used for selective and sensitive determination of parabens in aqueous samples.
\end{abstract}

(C) 2018 Elsevier B.V. All rights reserved.

\section{Introduction}

The esters of hydroxybenzoic acid, generally known as parabens

\footnotetext{
* Corresponding author.

E-mail addresses: mdemirkurt@gmail.com (M. Demirkurt), ahmeteroglu@iyte. edu.tr (A.E. Eroglu).

${ }^{1}$ Y.A. Olcer and M. Demirkurt contributed equally to this study.
}

are widely used as antimicrobial preservatives in food, cosmetic products and pharmaceuticals because of their broad antimicrobial spectra, chemical stability and low cost. Among them, methyl paraben and propyl paraben are the most commonly used together in products [1]. Despite widespread application of parabens as a harmless preservative because of low toxicity, several recent studies have revealed that parabens can show endocrine disrupting effects, a link between parabens and the risk of breast cancer, a 
relation with male infertility and other side effects [2]. It is known that various companies produce paraben-free products because of these facts. According to European Union, the commercial products should not contain higher concentrations than $0.4 \%(\mathrm{w} / \mathrm{w})$ for each paraben and a limit of $0.8 \%(\mathrm{w} / \mathrm{w})$ for total parabens [3]. Therefore, it is necessary to develop methods for the determination of parabens which can be emerging pollutants with adverse effects on human, animal and environment.

Several sample preparation methods including ultrasonic assisted extraction (UAE), supercritical fluid extraction (SFE), pressurized liquid extraction (PLE), solid phase extraction (SPE) [4-6], solid phase microextraction (SPME) [7,8], liquid phase microextraction (LPME), dispersive liquid-liquid microextraction (DLLME), stir bar sorptive extraction (SBSE), matrix solid phase dispersion (MSPD), and chromatographic methods including liquid chromatography (LC), gas chromatography (GC) [9-14], and capillary electrophoresis (CE) were reported for the determination of parabens in pharmaceutical formulation, in environmental matrices or in biologic samples [15-17]. Among them SPME is widely used as analysis technique for a great variety of compounds in environmental, food, biological, and pharmaceutical samples. However, commercial SPME fiber coatings suffer from lack of selectivity prior to GC applications. Moreover, analyte must be extracted from fiber coating with a proper desorption solvent prior to HPLC analysis and this step can destroy the fiber coating. To overcome these problems, many studies have been tried to develop solvent resistive and thermally stable SPME fiber coatings with high selectivity to the analytes of interest. Especially, fibers coated with molecularly imprinted polymers have gained attention due to their recognition ability to analyte molecule, low cost, mechanical and chemical stability [18].

In a recent study molecularly imprinted polymer coated fibers for solid-phase microextraction prepared in the presence of a single bifunctional monomer, N,O-bismethacryloyl ethanolamine, were used for extraction of parabens from environmental solid samples prior to LC-DAD analysis [19]. Preparation of a monolithic MIP by bulk polymerization with ethyl paraben as template molecule has been reported for a homemade SPME system [20]. MP, EP, PP were determined by GC-FID in three spiked soy samples with the recoveries of $95.48,97.86$, and $92.17 \%$ by using prepared MIP coated fibers.

In this work, electrospinning and MIP approaches were merged for the design of a reliable and efficient sorbent system. MIP microspheres entrapped in electrospun polystyrene fibers as the SPME fiber coating were developed for selective extraction and analyses of parabens for water system. The optimization parameters affecting the extraction and desorption of parabens were investigated. The validity of the proposed method was verified via spike recovery tests.

\section{Experimental}

\subsection{Materials and methods}

All chemicals and reagents were analytical reagent grade. Ultrapure water (18.2 M $\Omega$, Millipore Billerica, Massachusetts, USA) was used throughout the study. Glassware and plastics were washed firstly with acetone and then detergent and finally ultrapure water.

Methylparaben (99\% Methyl 4-hydroxybenzoate, MP), Propyl Paraben (99\% Propyl 4-hydroxybenzoate, PP), and Benzyl Paraben (99\% Benzyl 4-hydroxybenzoate, BP) were obtained from SigmaAldrich (St. Louis, MO, USA). Stock solutions (1000.0 $\mathrm{mg} \mathrm{mL}^{-1}$ ) of parabens were prepared in HPLC grade methanol (MeOH) (Sigma-Aldrich, St. Louis, MO, USA) monthly in amber bottles and stored at $-20^{\circ} \mathrm{C}$ in refrigerator. All standard solutions were prepared daily by appropriate dilution of the stock solution. The $\mathrm{pH}$ adjustments were done by using $1.0 \mathrm{M}, 0.1 \mathrm{M}, 0.01 \mathrm{M}$ of hydrochloric acid $(\mathrm{HCl})$ and sodium hydroxide $(\mathrm{NaOH})$ solutions. All standard and sample solutions were filtered through a $0.25 \mu \mathrm{m}$ cellulose acetate or polyamide membrane filter depending on the solvent system and degassed for $15.0 \mathrm{~min}$ in ultrasonic bath prior to HPLC analysis. The functional monomer methacrylic acid (MAA) and crosslinker trimethylolpropane trimethacrylate (TRIM) used in the MIP/NIP microsphere synthesis were purchased from Sigma-Aldrich (St. Louis, MO, USA), and the initiator 4,4'-Azobis (4cyanovaleric acid) (AIVN) were obtained from Alfa Aesar (Haverhill, Massachusetts, USA). In order to prepare electrospinable MIP/NIPSPME fiber coating, polystyrene (MW: $350 \mathrm{~kg} \mathrm{~mol}^{-1}$, Aldrich) was used as backbone and support material.

\subsection{Instrumentation}

Solution $\mathrm{pH}$ adjustment was performed with Ino Lab Level $1 \mathrm{pH}$ meter (Weilheim, Germany). To obtain effective mixing, IKA yellow line OS 5 basic orbital shaker (Staufen, Germany) was used.

Samples were analysed by using Agilent 1200 series HPLC equipped with Diode Array Detector (DAD) (Agilent Technologies, USA). An analytical column, C30 (YMC Caretenoid S-5 $\mu \mathrm{m}$, product code: CT99S05-2546WT, I.D.:25 cm $\times 4.6 \mathrm{~mm}$ ), was used for separation. Working standard solutions of each paraben $(0.025,0.050$ $0.10,0.25,0.50,1.0$, and $5.0 \mathrm{mg} \mathrm{L}^{-1}$ ) were prepared from their respective stock solutions. $\mathrm{MeOH}: \mathrm{H}_{2} \mathrm{O}$ mixtures in different compositions, several column temperatures, and flowrates were tried. The limit of detection (LOD) and limit of quantification (LOQ) were calculated.

MIP/NIP entrapped polystyrene nanofiber based SPME fiber coatings were obtained by using NanoScience electrospinning device (Inovenso, Basic system electrospinning, Yıldız Technical University, Teknopark, İstanbul).

\subsection{Synthesis of $M I P / N I P$ microspheres}

Firstly, MIP/NIP microspheres were synthesized following the same route described in our previous study [21]. The synthesis scheme was given in Fig. 1.

Briefly, the pre-polymer solution was prepared by mixing $1.0 \mathrm{mmol}$ benzyl paraben (template molecule) and $4.0 \mathrm{mmol}$ MAA (functional monomer) in $200.0 \mathrm{~mL}$ acetonitrile for one hour at room temperature.

Then, $16.0 \mathrm{mmol}$ TRIM (crosslinker) and 2.0\% ( $\mathrm{mol} / \mathrm{mol})$ AIVN (initiator) were added to reaction medium under $\mathrm{Ar}$ gas. The reaction system was sealed at $60^{\circ} \mathrm{C}$ for $8 \mathrm{~h}$. After polymerization, MIP microspheres produced were washed with $\mathrm{MeOH}$ and $\mathrm{MeOH}: \mathrm{H}_{2} \mathrm{O}$ mixture (80:20, pH 3.0, adjusted with acetic acid) in order to remove the template molecule. After complete removal of the template, the microspheres were dried in oven at $60^{\circ} \mathrm{C}$. The NIP microspheres were prepared following the same procedure except the addition of benzyl paraben.

\subsection{Preparation of molecularly imprinted membranes (MIMs), non imprinted membranes (NIMs) and electrospun SPME fiber coatings}

Firstly, the protective polymer coating of silica fibers was removed by immersing the fibers in acetone for one hour. After that, the surfaces of bare silica fibers were activated by using first $0.1 \mathrm{M} \mathrm{NaOH}$ and then $0.1 \mathrm{M} \mathrm{HCl}$ solution. Then, fibers were silylated for $30 \mathrm{~min}$ by exposing them to $10 \%(\mathrm{v} / \mathrm{v}) 3$-(methacryloxy) propyltrimethoxysilane solution in acetone at room temperature. Finally, the fibers were washed with methanol and dried prior to 


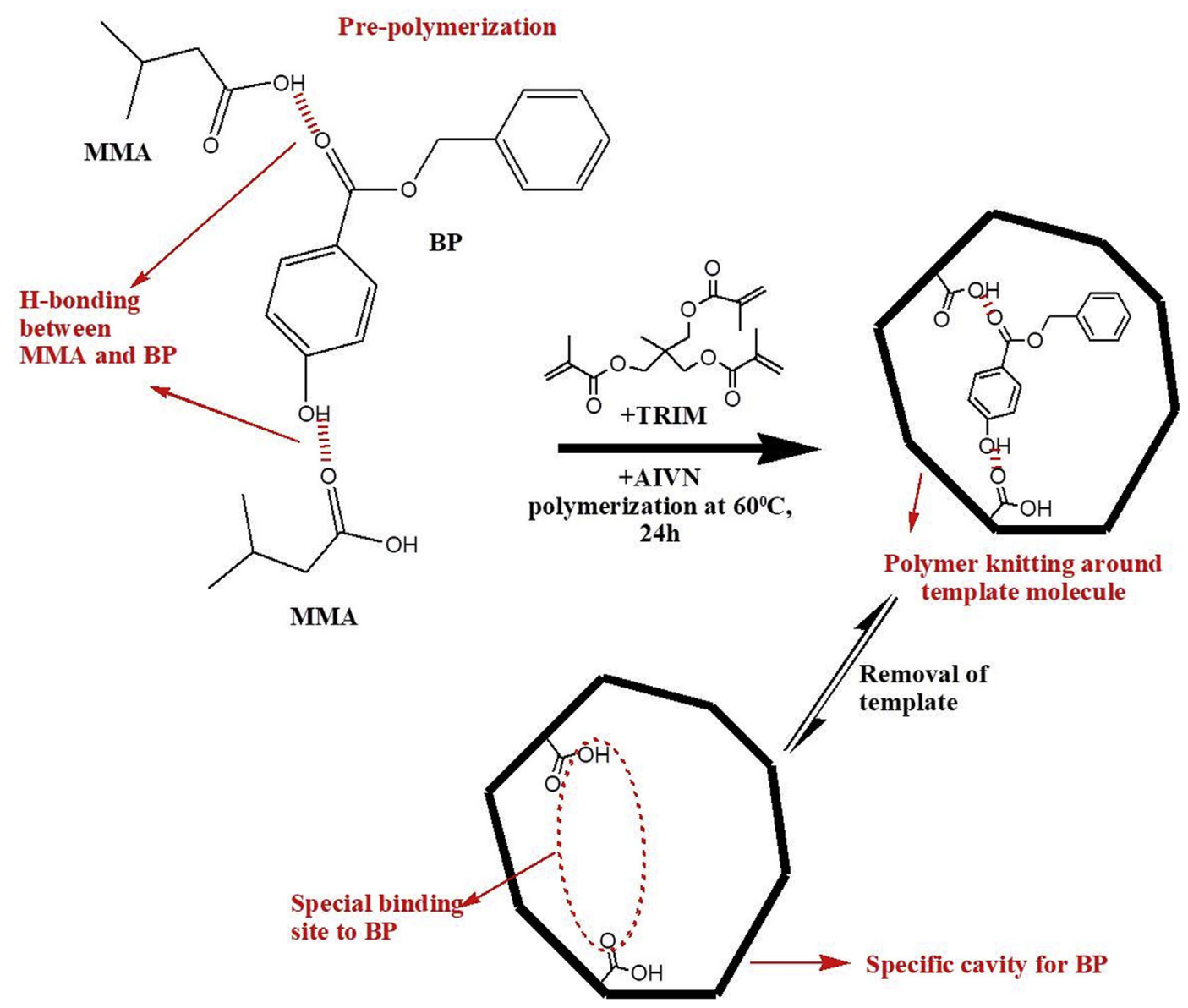

Fig. 1. Scheme of the MIP synthesis via co-polymerization of MAA and TRIM.

coating procedure.

For the electrospinning process, polystyrene was chosen as the backbone due to its intrinsic hydrophobicity and the inter- and intra-porous structure [22]. Firstly, 10\% (w/v) polystyrene solution in DMF was mixed with an equivalent amount of MIP or NIP particles (e.g., $1.0 \mathrm{~g}$ MIP or NIP was added for $1.0 \mathrm{~g}$ polystyrene in $10 \mathrm{~mL}$ DMF). After stirring for one hour, the syringe used in electrospinning apparatus was loaded with prepared solution and electrospinning process was initiated at $30.0 \mathrm{kV}$ potential differences with the flow rate of $1.0 \mathrm{~mL} \mathrm{~h}^{-1}$. A distance of $15.0 \mathrm{~cm}$ was chosen as the difference between the nozzle and the metal collector coated with aluminium foil. Silica fiber was attached on a mount made form a capillary tube in a way that $2.0 \mathrm{~cm}$ end of the fiber could have been exposed to electrospinning jet. The mount was attached on a rotating drum (300 rpm) which was placed between the metal collector and the nozzle and the fiber was coated with polystyreneknitted microspheres by exposing the fiber 10 or 20 times in a 1-sec inserts to electrospinning jet (Fig. 2). With this configuration, both MIP/NIP coated fibers and MIP/NIP membranes were prepared in the same run.

Coated fibers were conditioned at $110^{\circ} \mathrm{C}$. The MIMs and NIMs which were collected on aluminium foil were dried at room temperature.

\subsection{Characterization}

Presence of benzyl paraben cavities in MIMs was checked through the comparison of sorption capacities of MIMs and NIMs. For this purpose, SPE vacuum manifold (SUPELCO, 57030U) was used. Firstly, sample solutions were prepared from the stock solution of benzyl paraben at 1.0, 5.0, 10.0, 25.0, 50.0, 100.0 and $250.0 \mathrm{mg} \mathrm{L}^{-1}$ concentrations. Then, $1.0 \mathrm{~mL}$ solvent capacity columns were filled with $10.0 \mathrm{mg}$ of MIMs and NIMs in layers. Filled columns were washed with $20 \mathrm{~mL}$ of UPW in order to remove unretained MIPs/NIPs. Then, $10 \mathrm{~mL}$ sample solutions were passed through the columns and the effluents were collected for HPLC-DAD analysis.

After testing the existence of the cavities in MIMs, extraction ability of SPME coatings were examined. For this purpose 1.0, 5.0, $10.0,25.0,50.0,100.0$ and $250.0 \mathrm{ng} \mathrm{mL}^{-1}$ benzyl paraben solutions were prepared by dilution from the stock solution. The coated fibers were immersed into the solutions and stirred for $60 \mathrm{~min}$ on orbital shaker. Later, desorption was realized by immersing the fibers into $150 \mu \mathrm{L}$ of $\mathrm{MeOH}: \mathrm{H}_{2} \mathrm{O}$ mixture (80:20, pH 3.0, adjusted with acetic acid) for $30 \mathrm{~min}$. The effluents were analysed at HPLCDAD system.

\subsection{Optimization of parameters}

One of the most important parameters is the solution $\mathrm{pH}$. To understand the effect of $\mathrm{pH}$ on extraction of benzyl paraben by the prepared fibers, acidic, neutral and basic aqueous solutions of benzyl paraben were prepared. Extraction studies were performed at ambient conditions and the initial $\mathrm{pH}$ of solutions was adjusted separately to 3.0, 7.0 and 10.0 .

The adsorption and desorption kinetics are very significant parameters in SPME (equilibrium method); therefore various extraction and desorption times were examined for the prepared MIP fibers at pH 7.0. Extraction and desorption times of 5, 15, 30, 60, 


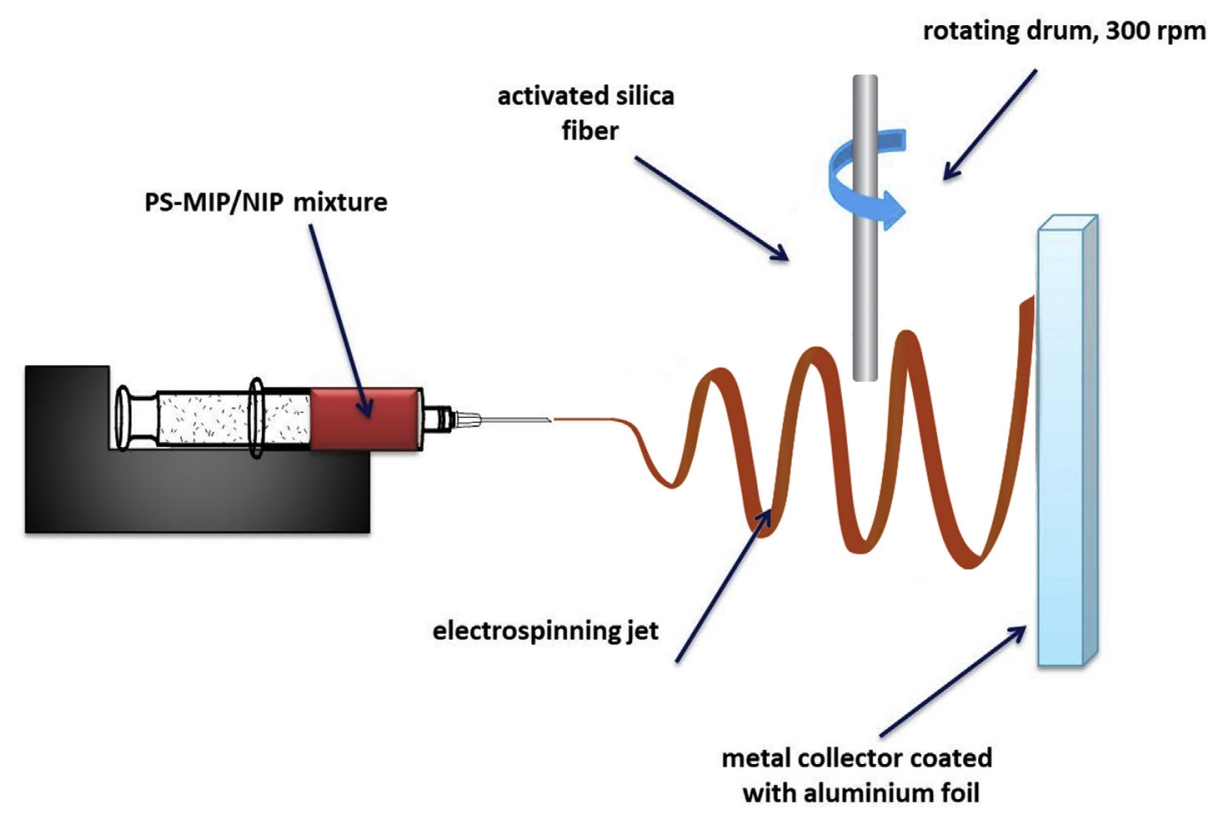

Fig. 2. Experimental set up for electrospinning and fiber coating process.

90, 120 min were tried.

The choice of desorption solvent is of great importance due to the possibility of breaking the hydrogen bonds between the template molecule and the active binding sites of MIP microspheres. Therefore, acetonitrile, methanol, methanol:HOAc (90:10) and $\mathrm{MeOH}: \mathrm{H}_{2} \mathrm{O}$ (80:20, pH 3.0, adjusted with acetic acid) were tried as desorption solvents.

The extraction ability of MIP-entrapped polystyrene-coated fiber was compared with commercially available SPME fibers including PDMS, PDMS/DVB/CAR and NIP fibers by using predetermined parameters.

Lastly, reusability of the sorbent was checked via repetitive sorption/desorption of benzyl paraben with proposed SPME method. After loading, the fiber was washed with $\mathrm{MeOH}: \mathrm{H}_{2} \mathrm{O}$ (80:20, pH 3.0, adjusted with acetic acid) solution, dried in an oven, and reused in the next sorption cycle. This procedure was repeated ten times.

\subsection{Cross selectivity}

For MIP synthesis, benzyl paraben was chosen as the template molecule due to its bulkier structure compared to structurally related methyl paraben and propyl paraben. It was assumed that the prepared fibers would be selective to these three compounds, but not to the other possible interferents. To check the validity of this assumption, $10 \mathrm{ng} \mathrm{mL}^{-1}$ of benzyl paraben, methyl paraben, propyl paraben, triclosan and triclocarban mixture were prepared and the SPME procedure were applied with the optimized experimental conditions.

\subsection{Method validation and sample analysis}

The MIP-entrapped fibers prepared were used to develop a method for determination of parabens by HPLC and UV detection under optimized conditions. The linearity, detection limit and precision of the analytical methodology were examined. The MIPSPME-HPLC method was applied for a linear range of $2.0-50.0 \mathrm{ng} \mathrm{mL}^{-1}$. The limit of detection (LOD) and limit of quantification (LOQ) were calculated.
Precision of the method was also examined by relative standard deviations of the peak areas for intra-day and inter-day extractions of the solution containing $10.0 \mathrm{ng} \mathrm{mL}^{-1}$ of each compound.

The developed MIP-SPME method was applied to analysis of tap, bottled and sea water samples spiked with $10.0 \mu \mathrm{g} \mathrm{L}^{-1}$ paraben mixture.

\section{Results and discussion}

\subsection{Synthesis of MIP/NIP microspheres}

Throughout the study, precipitation polymerization using noncovalent strategy was applied in which the polymerization was carried out in highly dilute solutions $(<5 \%(\mathrm{w} / \mathrm{v})$ monomers/porogen). This technique is proposed to provide more homogenous binding sites compared to bulk polymerization. It eliminates crushing and sieving steps that need to be carried out in bulk polymerization [23]. Also in our previous work [21], it was seen that MIP/NIP particles obtained with different polymerization methods can show difference in morphology. It was realized that the difference between the sorption capacity of spherical MIP and NIP was higher compared to monolithic MIP and NIP particles. In our work, highly diluted condition (1.25\% (w/v) monomers/porogen) was selected during the synthesis in order to obtain spherical MIP and NIP microspheres. These microspheres have the ability of being suspended in polystyrene solution which is useful at electrospinning process. Also, especially methacrylic acid was chosen as functional monomer due to its high selectivity to paraben species compared to 4-vinylpyridine (4-VP) [1]. The SEM image of synthesized MIP microspheres is given in Fig. 3.

\subsection{Preparation of molecularly imprinted membranes (MIMs), non imprinted membranes (NIMs) and electrospun SPME fiber coatings}

Electrospun fiber mats are good candidates for the entrapment of the spherical particles due to having micrometer sized interfibrillar spacing. The porous structure of individual fibers may further increase the efficiency of entrapment. Electrospinning of polystyrene from various polar solvents is a well-recognized system 


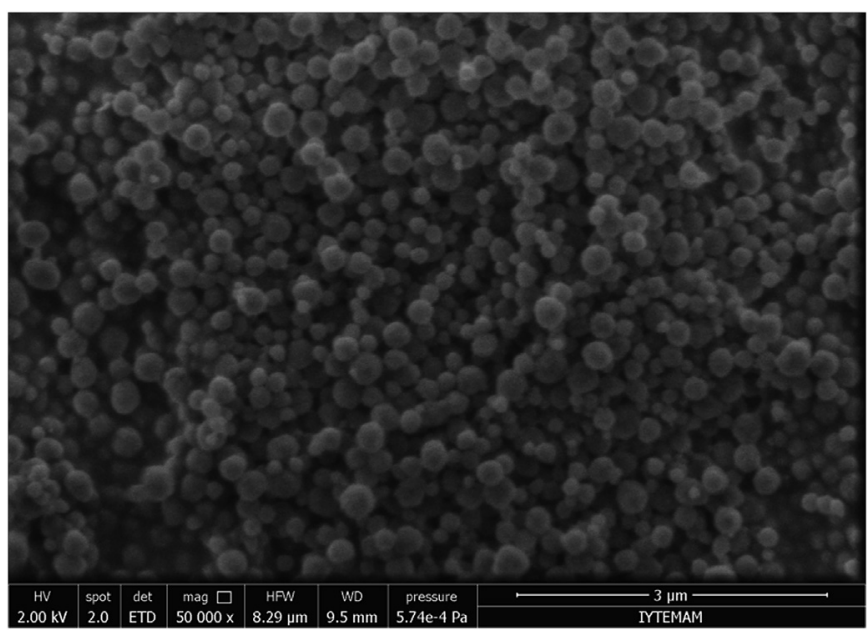

Fig. 3. SEM image of the MIP microspheres synthesized.

for the development of both interior and surface porosity of the individual filament that absolutely increases the surface area to volume ratio of system [22]. Humidity of the spinning environment was found to be the dominant parameter for the fabrication of porous electrospun polystyrene mats. Dimethyl formamide (DMF), solvent in our particular system, undergoes a liquid-liquid phase separation. Polystyrene is a hydrophobic polymer and can be dissolved in DMF; however, water is a nonsolvent. Therefore, polystyrene prefers to stay in DMF rather than being in water. Water evaporates before DMF due to its higher vapour pressure and leaves behind surface with interior porosity. This way, MIP and NIP submicron spheres are entrapped in polystyrene by electrospinning process.

Fig. 4 shows the SEM images of MIP microspheres entrapped in electrospun polystyrene fibers (MIMs) at various magnifications which were deposited on aluminum foil. It is clear that polymer microspheres stuck on the surface and inside each polystyrene nanofiber.

The mechanical stability of the materials was tested through crushing, dipping in nitrogen, washing with methanol and it was shown that these treatments did not leach out the microspheres from the fiber system and damage the morphology of the MIMs. Optimum thickness of coating on SPME fiber was obtained by trialand-error and coating process was applied several times in different trials to obtain the desired thickness. The morphology, homogeneity and thickness of the SPME fiber coating were examined by SEM after coating silica fibers 10 and 20 times (Fig. 5). The thickness measurements were made with ImageJ program and for the bare silica fiber it was calculated as $127.78( \pm 0.5) \mathrm{nm}$ whereas For 10 times- and 20 times-coated fibers, the thickness of the films was $156.91( \pm 2.2)$ and $466.81( \pm 3.4) \mathrm{nm}$, respectively. It can be realized from the SEM images that the polystyrene SPME fiber coating have MIP microspheres both inside and on the surface of each polystyrene fiber.

\subsection{Characterization}

Sorption ability of the MIMs and NIMs was checked via binding characteristic assay procedure as explained in Section 2.5. Fig. 6 shows the sorption properties of MIM and NIM. The ordinate, $\mathrm{Q}$ shows the amount (mmol) of benzyl paraben retained by $1.0 \mathrm{~g}$ of MIM or NIM. At low concentrations, there is no difference in the sorption capacities of the two membranes. However, after $250 \mathrm{mg} \mathrm{L}^{-1}$ there is a remarkable difference in sorption capacities. This difference remains unchanged for the higher concentrations although the capacity of both membranes decreases. The reason of this decrease may be the interaction of benzyl paraben molecules with each other at high concentrations. These results show the selectivity enhancement by the creation of specific binding (imprinted) sites in the sorbent by molecular imprinting.

The extraction ability of MIP-entrapped polystyrene fiber was examined by comparing with the NIP-entrapped polystyrene fiber which was prepared in the same way except for the addition of

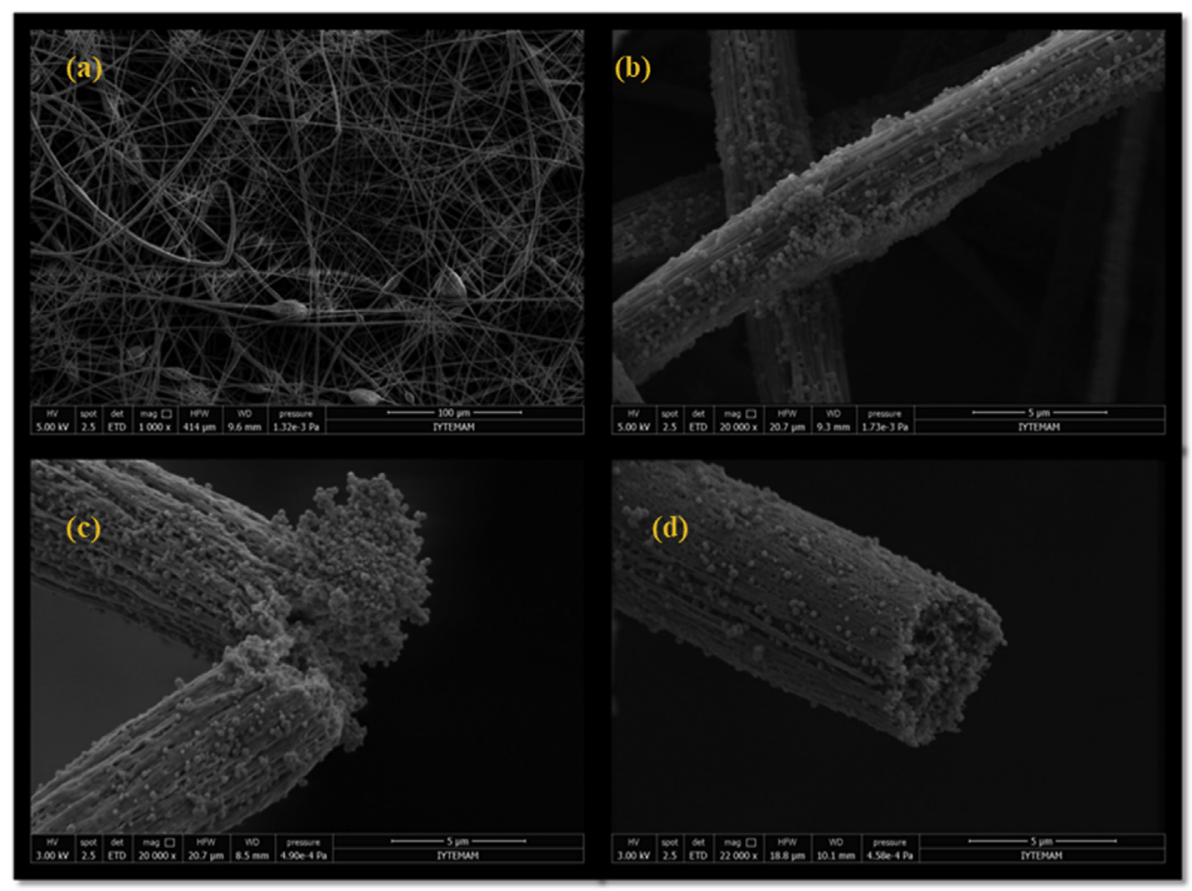

Fig. 4. SEM images of MIMs at (a) $1.000 \times$ (b) $20.000 \times$ (c) $20.000 \times$ and (d) $22.000 \times$ magnifications. 


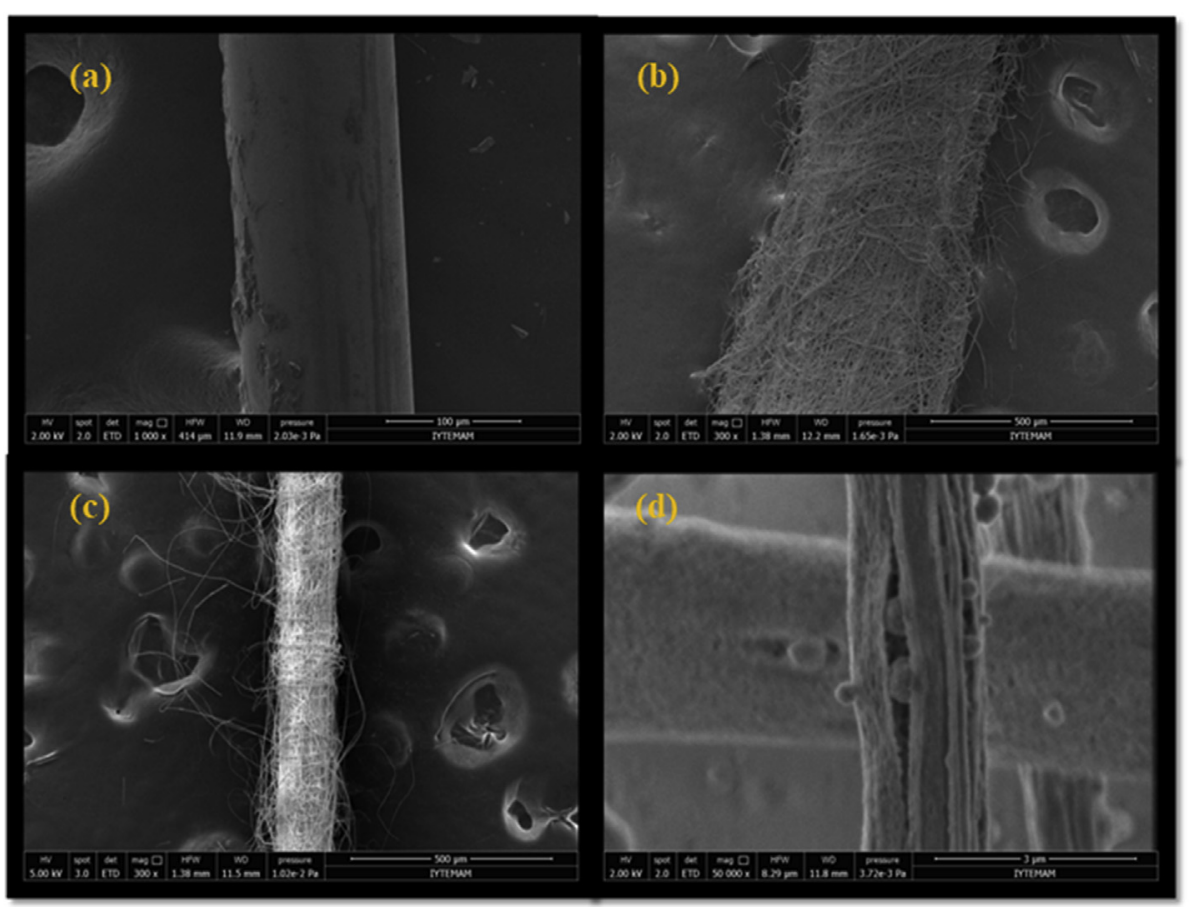

Fig. 5. SEM images of (a) the bare silica fiber, (b) MIP fiber coated 10 times, (c) MIP fiber coated 20 times and (d) the same fiber as in (c) with higher magnification.

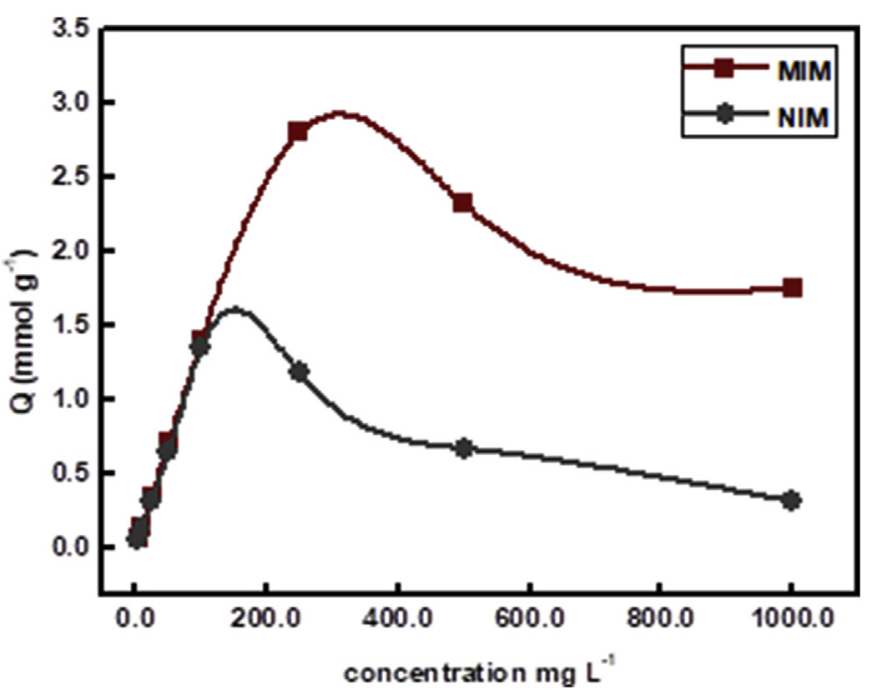

Fig. 6. Rebinding characteristics assay of MIMs and NIMs.

benzyl paraben as the template. Fig. 7 reveals the extracted amount of benzyl paraben on both SPME fiber coatings at different initial concentrations. It is clearly seen that the increase in initial concentration of benzyl paraben resulted in an increase in the extracted amounts for both fiber coating. However, the increase by MIP-entrapped polystyrene coated fiber was higher than the corresponding NIP-entrapped one. The reason must be the specific cavities created for benzyl paraben in the MIP-entrapped fiber. Also, polymer knitting formed around template molecule can provide non-specific interaction with template molecules and this property explains the sorption ability of the NIP-entrapped polystyrene fibers. These results again, demonstrate the selectivity enhancement against benzyl paraben by the specific imprinted sites on MIP.

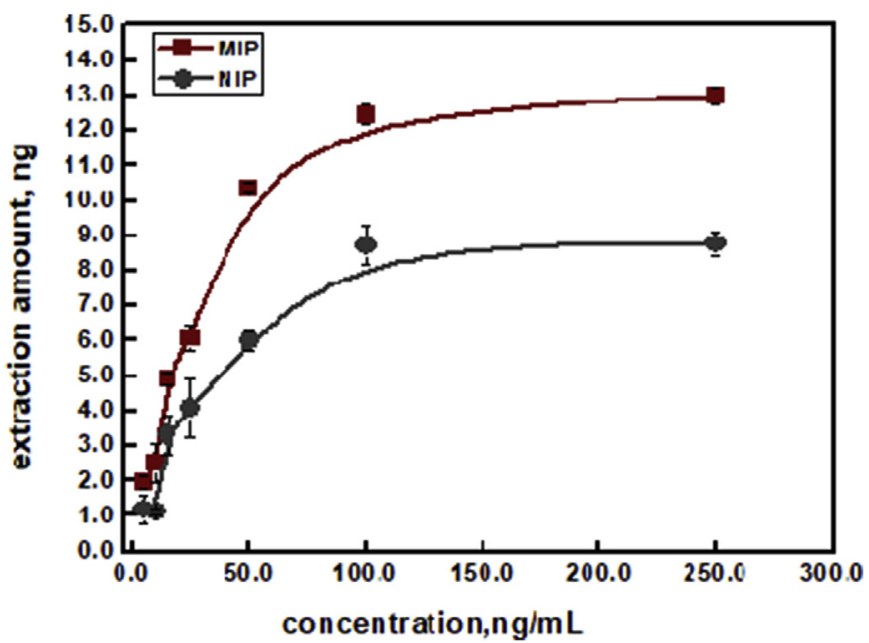

Fig. 7. Rebinding curves of BP with MIP and NIP coated fibers in spiked ultrapure water.

\subsection{Optimization of experimental parameters}

The first parameter optimized was the $\mathrm{pH}$ of working solution. As seen in Fig. 8 better extraction results were obtained at neutral and basic $\mathrm{pH}$ values than acidic conditions. The acid dissociation constant of benzyl paraben is 8.37. At pH 3 and 7, benzyl paraben is in its neutral form and can form easily $\mathrm{H}$-bonds with imprinted sites. It is expected to have better sorption-desorption results than basic conditions. However, the extracted amount at pH 3 is lower than expected for MIP. The reason must be the $\mathrm{pH}$ of the extraction solvent. As mentioned before, the extraction solvent is $\mathrm{MeOH}: \mathrm{H}_{2} \mathrm{O}$ (80:20, pH 3.0 adjusted with acetic acid). Here acetic acid destroys the H-bonding between MIP and the analyte molecule. The same $\mathrm{pH}$ value with working solution must prevent the extraction of 


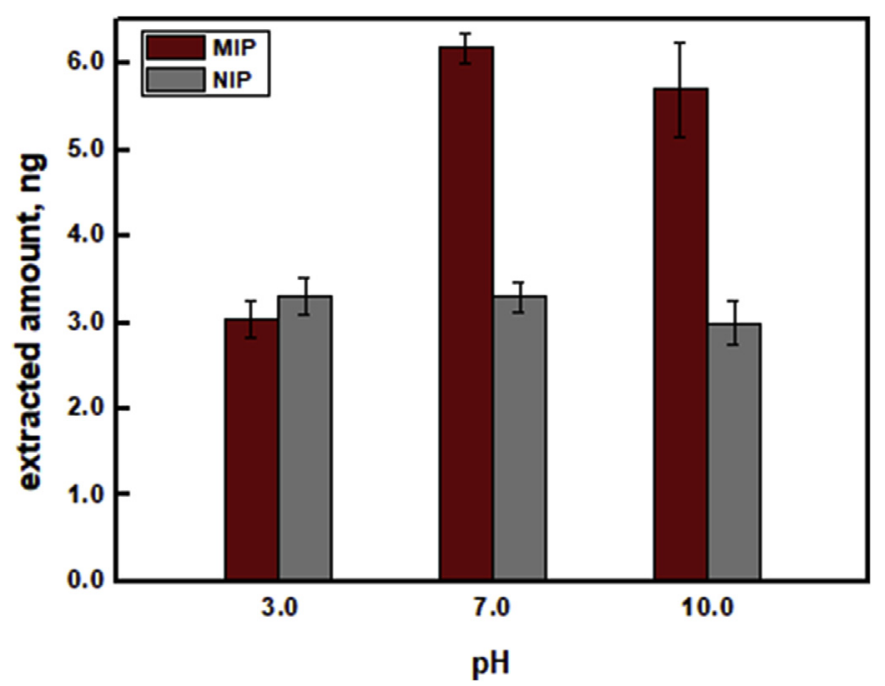

Fig. 8. The effect of solution pH on the extraction of benzyl paraben.

analyte. For $\mathrm{pH} 10$, both MIP and NIP has lower extraction value than $\mathrm{pH}$ 7. At basic conditions both MIP and benzyl paraben are deprotonated. This keeps them from making H-bond during sorption. Therefore, the extracted amount is lower than $\mathrm{pH} 7$.

Kinetic studies (Fig. 9) clearly show that extraction and desorption reached equilibrium at about $30 \mathrm{~min}$. Diffusion speed of

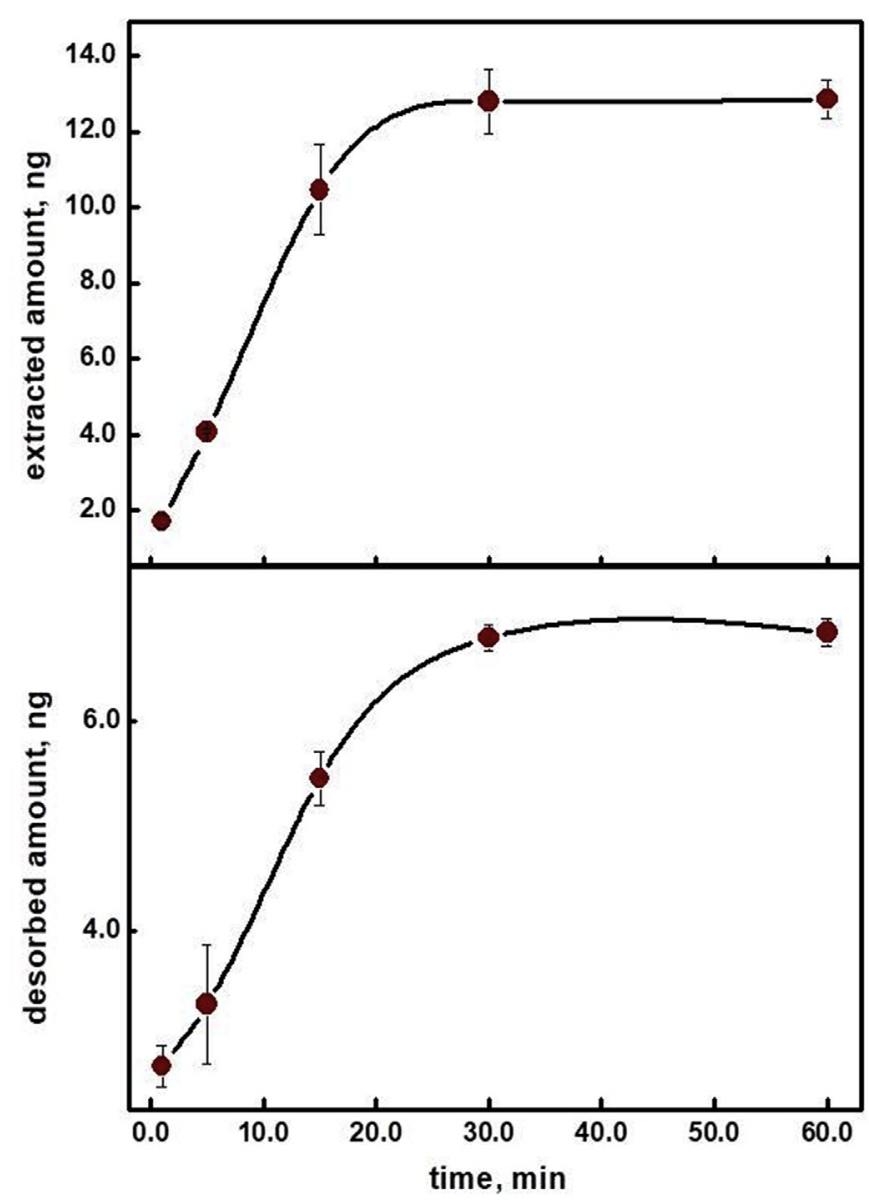

Fig. 9. Kinetics of (a) extraction and (b) desorption of benzyl paraben.

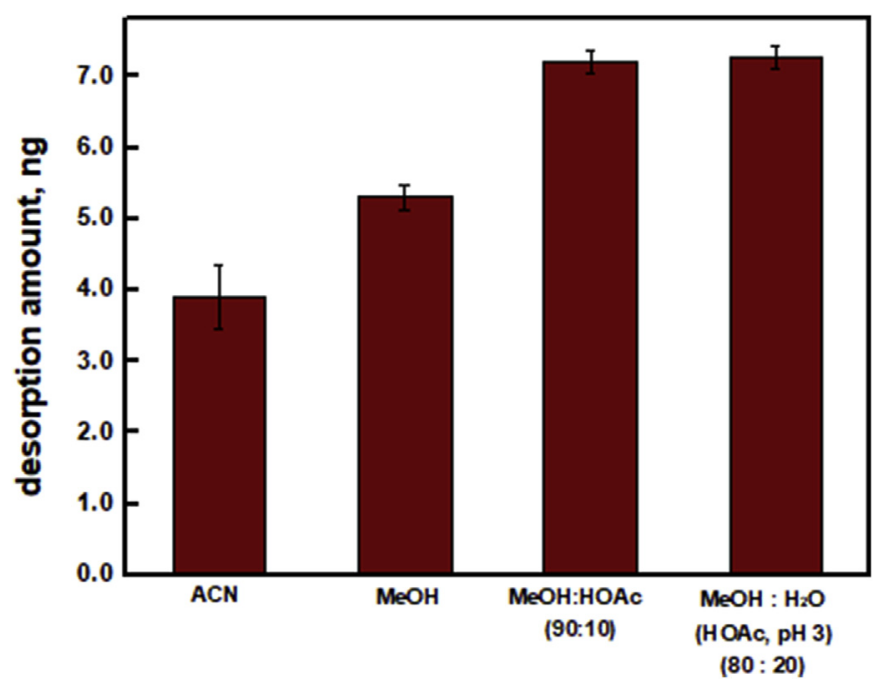

Fig. 10. Selection of desorption solvent.

the analyte species into or out of the coating is affected from the thickness of the sorbent layer.

Another parameter optimized in the SPME process is the kind and concentration of the desorption solvent. $\mathrm{MeOH}$ and water mixture exhibited highest desorption ability which could have reduced the hydrogen bonding between the analyte and the active sites of MIP microspheres. Finally, $\mathrm{MeOH}: \mathrm{H}_{2} \mathrm{O}$ mixture (80:20, pH 3.0, adjusted with acetic acid) was chosen as the desorption solvent to get rid of the template molecule, benzyl paraben, from MIP microspheres. This solution was also the mobile phase in HPLC-DAD analysis (Fig. 10). This was an important advantage that removed the solvent adjustment step prior to the introduction of the samples to HPLC. By this way, no baseline shift was observed during the chromatographic runs.

Finally extraction ability of the proposed sorbent (fiber) was compared with that of commercial fibers. Fig. 11 shows the extracted amount of benzyl paraben by MIP and NIP fibers and two other commercial SPME fibers at an initial concentration of $10.0 \mathrm{ng} \mathrm{mL} \mathrm{m}^{-1}$. The results show that MIP fiber developed in this study has higher extraction ability possibly because of specific hydrogen bonding interactions between the carboxylic groups in the MIP coating and hydroxyl moiety in benzyl paraben.

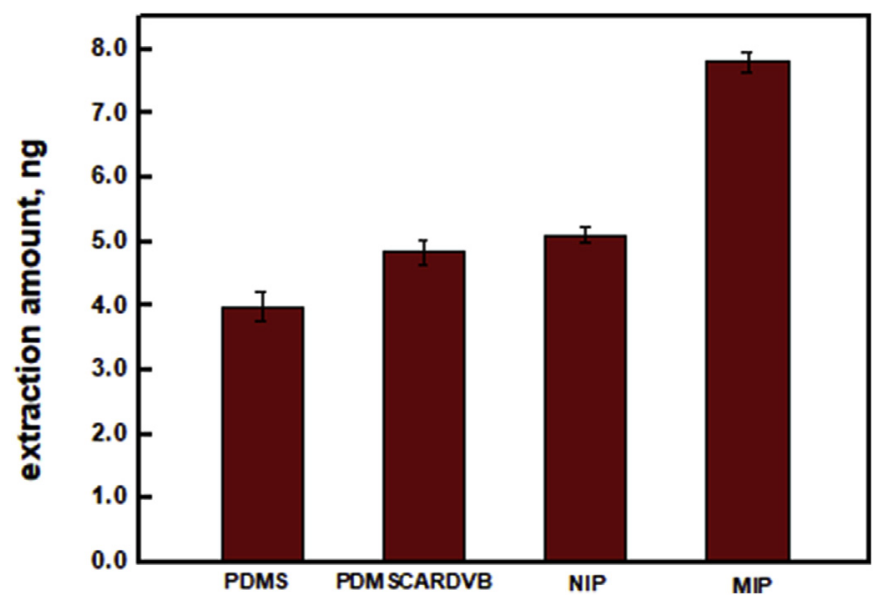

Fig. 11. The extracted amount of BP by the prepared MIP/NIP-coated fibers and by the commercial PDMS, PDMS/CAR/DVB. 


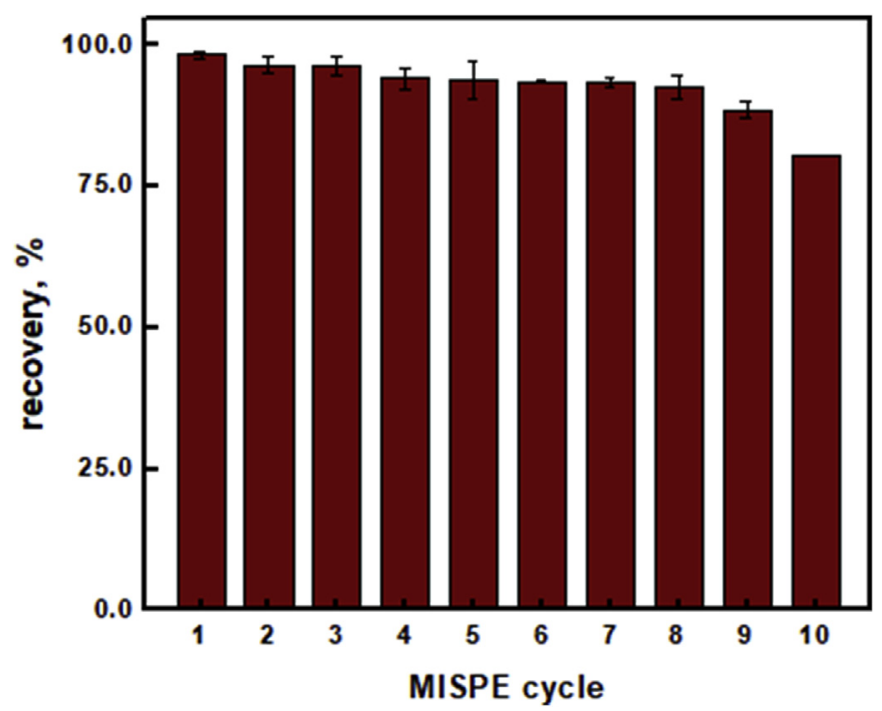

Fig. 12. Reusability of SPME fiber.

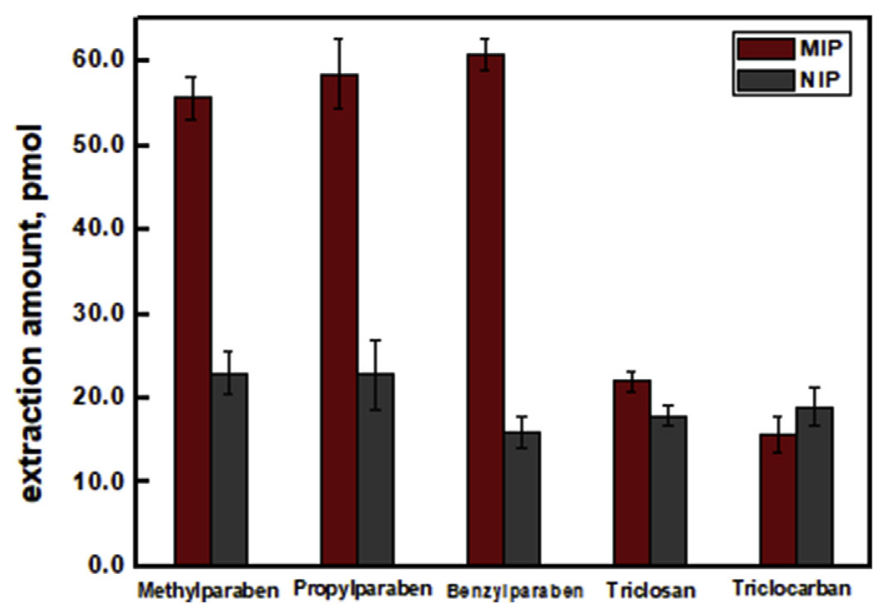

Fig. 13. Selectivity of the MIP-coated fiber to structurally-related compounds.
Reusability of MIP-coated SPME fibers was checked by subjecting the same fiber to consequent sorption/desorption cycles 10 times. The fiber shows higher than $90 \%$ sorption up to eighth cycle (Fig. 12). Then, the sorption decreases gradually due to the disruption of specific cavities during extraction. This number is low compared to the relatively higher reuse numbers of commercial fibers. However, this so-called disadvantage can be compensated by the selectivity of the SPME fibers prepared. Use of internal standard(s) is recommended in the analysis of real samples. Studies to improve the reusability of the MIP-coated SPME fibers are continuing.

\subsection{Cross selectivity}

The extraction ability of MIP- and NIP-entrapped polystyrene fiber for benzyl paraben was investigated in the presence of methyl paraben, propyl paraben, TCS and TCC as explained in Section 2.7. As seen in Fig. 13, MIP fiber showed high selectivity to all paraben species whereas other structurally related compounds, TCS and TCC were poorly extracted. Moreover, these results exhibit that the MIP microspheres prepared in this study have recognition ability to common structure of parabens. Among the paraben species examined, benzyl paraben was the most extracted.

\subsection{Method validation and sample analysis}

Under optimized conditions the MIP-SPME procedure was applied for the determination of paraben species. The LODs obtained for the parabens were in the range $0.26-0.29 \mathrm{ng} \mathrm{mL}^{-1}$. Also precision of the method was examined by relative standard deviations of the peak areas for intra-day and inter-day extractions of the solution containing $10.0 \mathrm{ng} \mathrm{mL}^{-1}$ of each paraben. For both inter- and intra-day study, relative standard deviations for paraben species were below $10 \%$ which shows the good performance of developed MISPME method (Table 1).

The methodology was also applied to tap, bottled and sea water samples spiked with $10.0 \mu \mathrm{g} \mathrm{L}^{-1}$ of paraben mixture and percent spike recoveries were calculated (Table 2). The results showed that the samples did not originally contain parabens in the detectable concentrations. Recoveries for the spiked samples were above $90 \%$ for all parabens. The proposed methodology could be used for selective determination of paraben species.

Table 1

Reproducibility of the methodology.

\begin{tabular}{|c|c|c|c|c|c|c|}
\hline \multirow[t]{2}{*}{ Compounds } & \multicolumn{4}{|c|}{ Calibration curve } & \multicolumn{2}{|c|}{$\operatorname{RSD}(n=6)$} \\
\hline & Slope & Intercept & $\begin{array}{l}\text { Linear range } \\
\left(\mathrm{ng} \mathrm{mL}^{-1}\right)\end{array}$ & $\begin{array}{l}\text { LOD } \\
\left(\mathrm{ng} \mathrm{mL} \mathrm{mL}^{-1}\right)\end{array}$ & Intra-day & Inter-day \\
\hline Methyl paraben & 2.374 & 0.580 & $2.0-50.0$ & 0.29 & 5.4 & 3.2 \\
\hline Propyl paraben & 2.134 & 0.370 & $2.0-50.0$ & 0.36 & 4.8 & 4.2 \\
\hline Benzyl paraben & 2.088 & 0.470 & $2.0-50.0$ & 0.26 & 4.2 & 4.6 \\
\hline
\end{tabular}

Table 2

Sample analysis.

\begin{tabular}{|c|c|c|c|c|c|c|}
\hline Compounds & $\begin{array}{l}\text { Bottled water } \\
\left(\mathrm{ng} \mathrm{mL}^{-1}\right)\end{array}$ & $\begin{array}{l}\text { Spiked } \\
\text { Bottled water } \\
\left(\mathrm{ng} \mathrm{mL}^{-1}\right)\end{array}$ & $\begin{array}{l}\text { Tap water } \\
\left(\text { ng } \mathrm{mL}^{-1}\right)\end{array}$ & $\begin{array}{l}\text { Spiked } \\
\text { Tap water } \\
\left(\mathrm{ng} \mathrm{mL}^{-1}\right)\end{array}$ & $\begin{array}{l}\text { Sea water } \\
\left(\text { ng } \mathrm{mL}^{-1}\right)\end{array}$ & $\begin{array}{l}\text { Spiked } \\
\text { Sea water } \\
\left(\mathrm{ng} \mathrm{mL}^{-1}\right)\end{array}$ \\
\hline Methyl paraben & ND & $94.8 \pm 1.2$ & ND & $92.2 \pm 0.8$ & ND & $99.7 \pm 0.4$ \\
\hline Propyl paraben & ND & $96.8 \pm 0.9$ & ND & $95.8 \pm 1.9$ & ND & $98.4 \pm 0.2$ \\
\hline Benzyl paraben & ND & $95.0 \pm 2.3$ & ND & $97.4 \pm 1.6$ & ND & $99.8 \pm 0.1$ \\
\hline
\end{tabular}

ND: Not Detected. 


\section{Conclusions}

In this study, MIP-entrapped electrospun polystyrene-coated SPME fiber with benzyl paraben as template was prepared for selective extraction of parabens. Its selectivity and extraction ability were compared with the commercial fibers and the corresponding NIP coated fiber. It was found that under optimized conditions, the prepared MIP-coated fiber showed better extraction ability than all the others. Also, the results revealed that MIP-coated fiber has better selectivity for benzyl paraben and the other structurallyrelated compounds, such as methyl and propyl paraben. Extraction efficiency of the prepared fibers for three parabens has been tested by spiking bottled, tap and sea water samples. The recoveries were between $94.8( \pm 1.2)$ and $96.8( \pm 0.9) \%$ for bottled, $92.2( \pm 0.8)$ and $97.4( \pm 1.6) \%$ for tap, and $98.4( \pm 0.2)$ and $99.8( \pm 0.7) \%$ for sea water at $10.0 \mu \mathrm{g} \mathrm{L}^{-1}$ levels. This MIPSPME HPLC method could be used for selective and sensitive determination of parabens by combination of two novel materials, electrospun fibers and MIP spheres.

\section{Acknowledgements}

The authors thank The Scientific and Technological Research Council of Turkey (TUBITAK) for the research project coded 114Z777. The authors are grateful also to IZTECH Center of Materials Research for the help in SEM analysis.

\section{References}

[1] L. Nunez, E. Turiel, A. Martin-Esteban, J.L. Tadeo, Molecularly imprinted polymer for the extraction of parabens from environmental solid samples prior to their determination by high performance liquid chromatographyultraviolet detection, Talanta 80 (2010) 1782-1788.

[2] S. Oishi, Effects of propyl paraben on the male reproductive system, Food Chem. Toxicol. 40 (2002) 1807-1813.

[3] C. Haman, X. Dauchy, C. Rosin, J.F. Munoz, Occurrence, fate and behavior of parabens in aquatic environments: a review, Water Res. 68 (2015) 1-11.

[4] A. Beltran, R.M. Marcé, P.A.G. Cormack, F. Borrull, Synthetic approaches to parabens molecularly imprinted polymers and their applications to the solidphase extraction of river water samples, Anal. Chim. Acta 677 (2010) 72-78.

[5] A. Azzouz, E. Ballesteros, Trace analysis of endocrine disrupting compounds in environmental water samples by use of solid-phase extraction and gas chromatography with mass spectrometry detection, J. Chromatogr. A 1360 (2014) 248-257.

[6] H.B. Lee, T.E. Peart, M.L. Svoboda, Determination of endocrine-disrupting phenols, acidic pharmaceuticals, and personal-care products in sewage by solid-phase extraction and gas chromatography-mass spectrometry, J. Chromatogr. A 1094 (2005) 122-129.

[7] D. Liu, N. Song, Y.C. Cheng, D.X. Chen, Q. Jia, Y.W. Yang, Pillarene functionalized polymer monolithic column for the solid-phase microextraction preconcentration of parabens, RSC Adv. 4 (2014) 49153-49160.

[8] J. Lopez-Darias, V. Pino, YJ. Meng, J.L. Anderson, A.M. Afonso, Utilization of a benzyl functionalized polymeric ionic liquid for the sensitive determination of polycyclic aromatic hydrocarbons, parabens and alkylphenols in waters using solid-phase microextraction coupled to gas chromatography-flame ionization detection, J. Chromatogr. A 1217 (2010) 7189-7197.

[9] S.R. Cao, Z.Y. Liu, L. Zhang, C.X. Xi, X.L. Li, G.M. Wang, R. Yuan, Z.D. Mu, Development of an HPLC-MS/MS method for the simultaneous analysis of six kinds of parabens in food, Anal. Meth. 5 (2013) 1016-1023.

[10] B. Saad, M.F. Bari, M.I. Saleh, K. Ahmad, M.K.M. Talib, Simultaneous determination of preservatives (benzoic acid, sorbic acid, methylparaben and propylparaben) in foodstuffs using high-performance liquid chromatography, J. Chromatogr. A 1073 (2005) 393-397.

[11] B.R. Ramaswamy, G. Shanmugam, G. Velu, B. Rengarajan, D.G.J. Larsson, GC-MS analysis and ecotoxicological risk assessment of triclosan, carbamazepine and parabens in Indian rivers, J. Hazard Mater. 186 (2011) 1586-1593.

[12] A.M. Peck, Analytical methods for the determination of persistent ingredients of personal care products in environmental matrices, Anal. Bioanal. Chem. 386 (2006) 907-939.

[13] I. Gonzalez-Marino, J.B. Quintana, I. Rodriguez, R. Cela, Simultaneous determination of parabens, triclosan and triclocarban in water by liquid chromatography/electrospray ionisation tandem mass spectrometry, Rapid Commun. Mass Spectrom. 23 (2009) 1756-1766.

[14] H.Y. Shen, H.L. Jiang, H.L. Mao, G. Pan, L. Zhou, Y.F. Cao, Simultaneous determination of seven phthalates and four parabens in cosmetic products using HPLC-DAD and GC-MS methods (vol 30, pg 48, 2007), J. Sep. Sci. 31 (2008), 1864-1864.

[15] C.Y. Piao, L.G. Chen, Y. Wang, A review of the extraction and chromatographic determination methods for the analysis of parabens, J. Chromatogr. B 969 (2014) 139-148

[16] J.A. Ocana-Gonazlez, M. Villar-Navarro, M. Ramos-Payan, R. Fernandez-Torres, M.A. Bello-Lopez, New developments in the extraction and determination of parabens in cosmetics and environmental samples. A review, Anal. Chim. Acta 858 (2015) 1-15.

[17] N. Cabaleiro, I. de la Calle, C. Bendicho, I. Lavilla, An overview of sample preparation for the determination of parabens in cosmetics, Trac-Trends Anal. Chem. 57 (2014) 34-46.

[18] L. Figueiredo, G.L. Erny, L. Santos, A. Alves, Applications of molecularly imprinted polymers to the analysis and removal of personal care products: a review, Talanta 146 (2016) 754-765.

[19] M. Diaz-Alvarez, S.P. Smith, D.A. Spivak, A. Martin-Esteban, Preparation of molecularly imprinted polymeric fibers using a single bifunctional monomer for the solid-phase microextraction of parabens from environmental solid samples, J. Sep. Sci. 39 (2016) 552-558.

[20] J. He, S. Chen, Y.L. Jiang, Y.Z. Shen, J. Zhu, H.L. Wei, H.X. Zhang, K. Lu, Preparation and selective recognition of a novel solid-phase microextraction fiber combined with molecularly imprinted polymers for the extraction of parabens in soy sample, J. Sep. Sci. 35 (2012) 308-314.

[21] Y.A. Olcer, M. Demirkurt, M.M. Demir, A.E. Eroglu, Development of molecularly imprinted polymers (MIPs) as a solid phase extraction (SPE) sorbent for the determination of ibuprofen in water, RSC Adv. (2017) 31441-31447.

[22] T. Isık, N. Horzum, U.H. Yıldız, B. Liedberg, M.M. Demir, Utilization of electrospun polystyrene membranes as a preliminary step for rapid diagnosis, Macromol. Mater. Eng. 301 (2016) 827-835.

[23] A. Beltran, F. Borrull, R.M. Marcé, P.A.G. Cormack, Molecularly-imprinted polymers: useful sorbents for selective extractions, TrAC Trends Anal. Chem. 29 (2010) 1363-1375. 